Revue musicale OICRM

\title{
Solliciter la voix des compositeurs. L'enquête de presse en France de la Première à la Seconde Guerre mondiale
}

\section{Cécile Quesney}

Volume 7, numéro 1, 2020

Écrits de compositeurs et espaces médiatiques

URI : https://id.erudit.org/iderudit/1069471ar

DOI : https://doi.org/10.7202/1069471ar

Aller au sommaire du numéro

\section{Éditeur(s)}

Observatoire interdisciplinaire de création et recherche en musique (OICRM)

ISSN

2368-7061 (numérique)

Découvrir la revue

Citer cet article

Quesney, C. (2020). Solliciter la voix des compositeurs. L'enquête de presse en France de la Première à la Seconde Guerre mondiale. Revue musicale OICRM, 7(1), 62-77. https://doi.org/10.7202/1069471ar
Résumé de l'article

Variante générique de l'interview, l'enquête journalistique connaît un grand succès dès son apparition en France à la fin du XIX ${ }^{\mathrm{e}}$ siècle. Lorsque la musique constitue le sujet de l'enquête, les journalistes sollicitent principalement des compositeurs qui apposent ainsi leur signature autorisée à leurs articles. L'enquête est donc un genre qui met les créateurs au-devant de la scène médiatique, mais qui implique aussi que leur discours leur échappe : les réponses sont publiées et commentées par un journaliste et sont insérées dans un véritable feuilleté polyphonique puisque l'enquêteur rapporte les propos d'une série de personnalités qui peuvent aussi profiter du sondage pour débattre entre pairs. En procédant par coups de sonde dans ce très vaste corpus, cet article propose d'examiner les enjeux et les déclinaisons d'un genre littéraire qui a su susciter l'intérêt des journalistes et des compositeurs jusqu'aux lendemains de la Seconde Guerre mondiale. 


\title{
Solliciter la voix des compositeurs. L'enquête de presse en France de la Première à la Seconde Guerre mondiale
}

\author{
Cécile Quesney
}

\begin{abstract}
Résumé
Variante générique de l'interview, l'enquête journalistique connaît un grand succès dès son apparition en France à la fin du XIXe siècle. Lorsque la musique constitue le sujet de l'enquête, les journalistes sollicitent principalement des compositeurs qui apposent ainsi leur signature autorisée à leurs articles. L'enquête est donc un genre qui met les créateurs au-devant de la scène médiatique, mais qui implique aussi que leur discours leur échappe : les réponses sont publiées et commentées par un journaliste et sont insérées dans un véritable feuilleté polyphonique puisque l'enquêteur rapporte les propos d'une série de personnalités qui peuvent aussi profiter du sondage pour débattre entre pairs. En procédant par coups de sonde dans ce très vaste corpus, cet article propose d'examiner les enjeux et les déclinaisons d'un genre littéraire qui a su susciter l'intérêt des journalistes et des compositeurs jusqu'aux lendemains de la Seconde Guerre mondiale.
\end{abstract}

Mots clés : compositeurs ; enquête ; France ; presse ; $\mathrm{xx}^{\mathrm{e}}$ siècle.

\begin{abstract}
A variant of interview, the "journalistic investigation" is very successful as soon as it appears in late $19^{\text {th }}$ century France. When music is the object of the investigation, journalists mainly appeal to composers who can then append their authorized signature to their articles. While putting creators at the front of the media stage, this genre also implies that the composers' discourse is beyond their full control: their answers are published and commented on by a journalist and then inserted into a polyphonic millefeuille, since the journalist reports the words of different personalities who get advantage of the survey to fuel the debate among peers. Considering some cases in this large corpus, this article gives an account of the issues raised by this literary genre takes and of the different forms it takes up to 1950 .
\end{abstract}

Keywords: composers; France; press; survey; $20^{\text {th }}$ century. 
L'enquête menée auprès de personnalités du monde culturel et artistique apparaît en France au début des années $1890^{1}$. Publiée dans les journaux et les revues, elle est diligentée par un journaliste qui questionne successivement plusieurs personnes sur un même sujet et publie leurs réponses au sein d'un ou plusieurs articles. D'abord menée auprès des hommes de lettres, l'enquête constitue un avatar de la fameuse "visite au grand écrivain "(Carbonnel 2004a). Elle adopte ainsi la forme d'un entretien retranscrit - que nous pouvons appeler enquête entretien - mais peut aussi se présenter sous la forme d'un questionnaire écrit auquel les enquêtés répondent par lettre - forme que nous pouvons qualifier d'enquête épistolaire. L'enquête constitue dans les deux cas un sous-genre de l'interview qui est elle-même une déclinaison du reportage $^{2}$.

Dans le monde de la musique, comme dans le monde des lettres, c'est avant tout aux auteurs que les journalistes adressent leurs enquêtes. Marie Carbonnel a identifié cinq catégories d'enquêtes aux écrivains menées au début du Xx siècle (ibid.) : 1) les enquêtes portant sur le métier d'écrivain, 2) celles dédiées à tel aspect de la création contemporaine, 3) les enquêtes biographiques menées à l'occasion d'une distinction, d'un décès ou d'un anniversaire, 4) celles portant sur les tendances actuelles d'un genre littéraire, et enfin 5) les enquêtes " généralistes " visant à dresser un bilan et des perspectives pour l'avenir de la littérature. Si le premier type de sondage est peu exploité dans les enquêtes aux musiciens - sans doute car le métier de compositeur risque de susciter un discours trop technique -, les autres catégories sont très bien représentées, en particulier les enquêtes portant sur un courant contemporain ou un genre artistique. S'inscrivant dans l'actualité musicale, les questionnaires sur les tendances de la musique interrogent en effet les acteurs du milieu sur les développements artistiques (comme la rupture avec la tonalité ou l'apport du jazz), mais aussi technologiques (disque, radio, cinéma). Elles répondent également à l'actualité immédiate : l'anniversaire de tel compositeur ou de telle création, la reprise d'une œuvre célèbre, les politiques d'enseignement et de financement de la musique ou encore la situation de guerre sont ainsi l'occasion d'interroger les compositeurs sur des questions artistiques, mais aussi, quelques fois, culturelles et politiques. Enfin, les questions générales sur l'évolution de la musique se retrouvent elles aussi jusqu'au milieu du siècle : on demande alors aux compositeurs de se prononcer sur les tendances de la création musicale et sur l'avenir de la musique. Le questionnaire est quelque fois centré sur un genre, en particulier l'opéra, dont la crise, diagnostiquée dès le début du siècle, ne cesse de faire couler de l'encre.

L'enquête de presse connaît un développement sans précédent à partir de la fin du $\mathrm{XIX}^{\mathrm{e}}$ siècle et on peut estimer à plusieurs centaines le nombre d'enquêtes sur la musique

1 Je remercie vivement Michel Duchesneau et Benoit Haug de m'avoir transmis une importante liste de sources issues du dépouillement de trois revues spécialisées : La Revue musicale, Le Courrier musical et Le Guide du concert (Duchesneau 2016, Haug 2010).

2 L'interview, "forme hybride, à double voix et double signature ", connaît une vogue considérable depuis sa naissance aux États-Unis, contribuant au développement d'un journalisme d'actualité destiné à un large public (Seillan 2011, p. 1028). 
parues dans les journaux et revues françaises dans la première moitié du $\mathrm{Xx}^{\mathrm{e}}$ siècle ${ }^{3}$. Les propos collectés par chaque journaliste ${ }^{4}$ fournissent souvent la matière à une série d'articles. La publication des résultats peut alors se déployer sur plusieurs mois, selon le succès du sondage et l'importance que lui accordent le journal et l'enquêteur. Ces « réponses à une enquête » constituent donc un corpus d'une richesse exceptionnelle, difficile à traiter dans sa totalité ${ }^{5}$.

Si les enjeux biographiques et médiatiques de l'interview du début du siècle ont récemment été mis en lumière (Duchesneau 2016, Campos 2016), les spécificités de l'enquête et son évolution de la Première à la Seconde Guerre mondiale restent à explorer. L'enquête est un genre qui met les créateurs au-devant de la scène médiatique mais implique aussi que leur discours leur échappe : les réponses sont publiées sans droit de regard et sont insérées dans un véritable « feuilleté de voix » (Thérenty 2009, $\S 30$ ) puisque l'enquêteur rapporte et commente les propos d'une série de personnalités qui peuvent aussi profiter du sondage pour débattre entre pairs. On peut donc s'interroger sur les fonctions de l'enquête et plus particulièrement sur les stratégies des enquêteurs et des compositeurs sollicités. L'importance et la permanence de ce genre invitent également à examiner son rapport au milieu musical : 1'enquête a-t-elle révélé ou nourri les questionnements ou les débats des musiciens ? A-t-elle évolué au fil du temps et comment a-t-elle été perçue ? Le présent article, qui ne vise pas à l'exhaustivité, propose de répondre de façon provisoire à ces questions à partir d'un corpus d'une vingtaine d'enquêtes parues dans divers périodiques parisiens, de la Première Guerre mondiale aux années qui ont suivi la Libération ${ }^{6}$. Publiés grâce au concours de journalistes, de critiques musicaux et de compositeurs plus ou moins reconnus, les articles pris en compte ont été retenus dans l'optique de présenter le genre dans toute la variété de ses formes, de ses supports et de ses contenus. Une importante enquête du journaliste Pierre Maudru dans Comodia en 1928 (19 articles publiés en une du journal) servira en quelque sorte de point d'ancrage dans la mesure où elle résume plusieurs caractéristiques des enquêtes de la période dans la presse culturelle ${ }^{7}$.

3 Voir la liste partielle établie par Rémy Campos pour la période 1895-1932 (Campos 2016, p. 157-159) ainsi que les sources rassemblées dans la base de données du Laboratoire Musique Histoire et Société (LMHS) de l'oicRM (catégorie "Enquêtes et entrevues avec des musiciens »), http://1mhsbd.oicrm.org/, consulté le 15 juillet 2019.

4 Les résultats publiés vont de quelques réponses à près de 80 propos rapportés pour une même enquête. Le très grand nombre de réponses (79) à l'enquête sur « la musique et le sport " publiées dans Le Guide du concert en 1924-1925 semble être un cas assez exceptionnel. Sur cette enquête, voir l'étude de Federico Lazzaro (2017).

5 La numérisation progressive de nombreux corpus de presse offre toutefois de plus en plus de d'outils pour le dépouillement.

$6 \quad$ Il s'agit d'enquêtes publiées dans les journaux et revues suivantes : Le Cri de Paris, La Renaissance politique, littéraire et artistique, La Revue musicale, Le Courrier musical, Le Guide du concert, Comodia, Paris-Midi, Musique, Le Ménestrel, Contrepoints et Arts. Un dépouillement systématique de ces périodiques doit encore être réalisé.

$7 \quad$ Cette enquête au long cours porte sur une question très générale (" L'avenir de la musique française ") qui mobilise de nombreux acteurs. Elle adopte une forme mixte (enquête entretien et enquête épistolaire) et révèle les principales préoccupations du milieu musical de la fin des années 1920. 
Ces différentes sources permettront ainsi d'explorer les enjeux et les modalités de l'enquête de presse aux compositeurs, d'abord du point du des enquêteurs, puis de celui des enquêtés.

\section{L'ENQUÊTEUR AU TRAVAIL}

L'enquête est présentée comme un outil d'information en ce qu'elle réunit diverses opinions sur un sujet d'actualité. Elle est aussi pour le journaliste un moyen de s'associer à des noms prestigieux et par là-même d'élargir potentiellement son lectorat. L'enquêteur qui signe en bas des textes peut ainsi s'entourer de personnalités reconnues (présentées en titre, en sous-titre ou en incipit) et tirer avantage d'une association basée sur le bénéfice mutuel de la publication.

La collecte est fréquemment assortie d'un effort d'ordonnance, de classement et de synthèse des réponses recueillies : c'est l'occasion pour l'enquêteur de mettre en valeur ses connaissances en matière de musique (lorsqu'il en a, car il n'est pas toujours spécialiste du domaine), de déceler des tendances et de positionner les compositeurs les uns par rapport aux autres. L'opération est facilitée par le fait qu'il formule généralement une liste de questions afin de guider les personnalités sondées : dans ce cas le questionnaire est souvent publié et accompagné d'une introduction ainsi que de remarques préalables. Dans son enquête épistolaire sur « les applaudissements au concert " menée en 1932-1933, Le Guide du concert indique ainsi en préambule, après avoir introduit son sujet et avant de détailler son questionnaire : "Sans vouloir influencer nos lecteurs en faveur d'une opinion et point même de la nôtre, que nous aurons la discrétion de taire, il nous semble qu'il y aurait lieu de fixer les points suivants » ([Bender] 1932).

De fait, le journaliste se présente généralement en observateur qui ne fait que recueillir les voix. Mais s'il se défend de prendre parti, en réalité sa position est souvent loin d'être neutre ${ }^{8}$. De plus, les enquêtes s'adressent d'abord à des compositeurs reconnus, souvent âgés, et qui occupent une position institutionnelle. Les compositeurs plus jeunes ou en voie de consécration sont généralement moins présents. Ainsi, seules 5 des 18 personnalités consultées dans le cadre de l'enquête de Comodia de 1928 sur "L'avenir de la musique " ont moins de 40 ans, tandis que 11 d'entre elles ont dépassé la soixantaine. L'enquêteur, Pierre Maudru, précise bien dans l'un de ses articles qu'il s'est « imposé comme règle d'accueillir avec la même impartialité l'expression des tendances les plus différentes, partant des diverses générations actuelles "(Maudru 1928h, p. 1). Le journaliste favorise pourtant les

Le journaliste Pierre Maudru y publie les réponses de 17 hommes, presque tous compositeurs, du 5 février au 26 mai 1928 : Gabriel Pierné, Henri Rabaud, Vincent d'Indy, Arthur Honegger, Georges Hue, Paul Dukas, Florent Schmitt, André Messager, Philippe Parès, Georges Van Parys, Alfred Bruneau, Paul Witkowski, Guy Ropartz, Hans Sachs, Paul Vidal, Louis Aubert et Darius Milhaud. Elle est conclue par un article en deux parties du critique Pierre Lalo.

8 Comme l'a montré Campos, l'enquête de Musica sur « les droits respectifs des compositeurs et des librettistes » lancée en 1909 à l'occasion d'un conflit entre Maurice Maeterlinck et Jacques Février prenait ainsi très clairement le parti des compositeurs (Campos 2016, p. 159-160). 
compositeurs âgés qui pour la grande majorité en appellent aux valeurs de la tradition musicale savante (le beau associé au système tonal, l'étude des maîtres et le travail désintéressé) afin de discréditer les travaux de jeunes musiciens qu'ils accusent d'amateurisme et d'arrivisme.

L'enquête de Maudru offre une véritable mise en scène de cette opposition générationnelle : recréant l'impression d'une discussion sur le vif, les paroles rapportées sont caractérisées par un style oral et relâché propre au genre de l'interview, qui vise ainsi à s'approcher au plus près de la personnalité du créateur ${ }^{9}$. Mais il s'agit aussi de présenter ce créateur dans son univers familier : en décrivant son cadre de vie, le journaliste propose ainsi au lecteur d'entrer dans l'atelier du compositeur et de percer sa psychologie (Carbonnel 2004b, p. 37). Cette attention portée à la maison ou au lieu de travail du créateur, déjà présente dans les entretiens d'écrivains et d'artistes du début du siècle ${ }^{10}$, se retrouve dans plusieurs articles de Maudru. Le texte consacré à Henry Rabaud, compositeur et directeur du Conservatoire, s'ouvre ainsi par une description du bureau dans lequel a lieu la rencontre :

Le vaste bureau directorial, situé au-dessus des salles d'examen, domine la cour silencieuse du Conservatoire. Aux murs, quelques photographies ou peintures de maîtres illustres : sous la haute fenêtre, au jour tamisé par des rideaux, s'ouvre un « demi-queue » chargé de partitions. Le cadre est sévère ; l'hôte est souriant.

Sur la table sont éparpillés deux ou trois actes manuscrits. La couverture en est neuve. Qui donc avait annoncé dans la presse que le compositeur de Marouf, trop absorbé par ses fonctions officielles, renonçait à produire? Ses admirateurs s'étaient émus de cette information. Or, ne serait-ce point un nouveau livret que j'aperçois? Serait-il aussi de M. Lucien Népoty qui fut déjà deux fois le collaborateur de M. Henri Rabaud pour Le Premier Glaive et pour Marouf?

M. Henri Rabaud élude nos questions et se défend d'avoir des projets (Maudru 1928b, p. 1).

On le voit, cette description du bureau est aussi un portrait psychologique de l'homme : Rabaud est un directeur respecté et dévoué qui veille sur ses élèves ; il est un gardien de la tradition des "maîtres illustres ", mais aussi un musicien productif. Concentré sur sa noble tâche de former les jeunes générations de musiciens, il n'en néglige pas moins la composition - le journaliste profite d'ailleurs de la mention des manuscrits pour susciter la curiosité du lecteur sur un nouvel ouvrage en cours.

\footnotetext{
9 Seuls trois compositeurs répondent à l'enquête par lettre. Ceci est probablement dû au fait qu'ils résident en province.

10 La première grande entreprise de ce type avait été menée en 1891 par Jules Huret, qui avait reproduit les propos de 64 écrivains dans L'Écho de Paris. Cette vaste enquête s'inspirait de la « visite au grand écrivain ", mais proposait une version plus séculière de ce rituel de la rencontre dans la demeure du créateur (Carbonnel 2004b, p. 37). Les artistes sont également sollicités depuis le XIX ${ }^{\mathrm{e}}$ siècle comme l'ont montré les travaux de Rachel Esner sur les visites d'ateliers de peintres et de sculpteurs rapportées dans la presse illustrée (voir, par exemple, Esner 2013). Les compositeurs, moins systématiquement connus du public, semblent plus rarement consultés chez eux. Jules Massenet est de ce point de vue un cas particulier : véritable vedette, il est à l'honneur dans la revue Musica où il fait l'objet d'une série d'entretiens et de reportages dans les années 1900 (Campos 2016, p. 161-164).
} 
L'usage d'accessoires, récurrent dans les enquêtes aux écrivains, peut aussi permettre de « décoder » la personnalité de l'auteur interrogé (Carbonnel 2004a, § 16) : c'est le cas par exemple de l'article de la même enquête consacré aux réponses de Florent Schmitt (Maudru 1928f). Le constat de l'impuissance des jeunes compositeurs est illustré par l'échec répété de Schmitt pour allumer sa pipe : "Attristé par cette constatation, M. Florent Schmitt bourre une pipe et rate successivement quatre ou cinq allumettes, dont le bois casse ou le phosphore ne prend pas ». Et Schmitt de reprendre un peu plus tard : "Chez nous [...] la flamme manque... Puis, comme il a fait une nouvelle tentative infructueuse pour allumer sa pipe, M. Florent Schmitt ajoute en souriant - Elle manque jusque dans les allumettes ! " Les allumettes reviennent à plusieurs reprises ponctuer l'entretien : elles figurent l'opiniâtreté et le caractère provocateur du compositeur, mais permettent aussi de montrer qu'il est un homme comme les autres. Maudru conclut l'entretien par ces mots : "Un silence. M. Florent Schmitt, qui est enfin parvenu à allumer sa pipe, tire avec satisfaction deux ou trois bouffées, réfléchit un instant et dit, en manière de conclusion : "Puis tout cela ne serait rien si nous avions de bonnes allumettes ${ }^{11}$ !" » (ibid., p. 1-2)

Maudru, qui est également réalisateur et scénariste, multiplie ainsi les anecdotes et les effets dramaturgiques ${ }^{12}$. Comme dans le cas des interviews d'écrivains, il ne s'agit pas uniquement de proposer une transcription du contenu de l'échange, mais aussi de susciter la curiosité du lecteur en lui offrant des détails amusants et un texte accrocheur. Le récit, qui alterne entre style direct et style indirect libre se veut vivant et divertissant. La langue peut être simple voire familière ${ }^{13}$, afin de montrer l'homme " tel qu'il est ». L'entretien de Schmitt dévoile ainsi le franc parler d'un musicien qui n'hésite pas à attaquer ses pairs de façon dégradante, comme en témoigne son jugement sans appel à propos de jeunes compositeurs : " ils sont bons à mettre au cabinet» (ibid., p. 2).

Choisir de publier de tels propos montre que le journaliste cherche aussi, parfois, à susciter la polémique et à exciter les conflits dans le milieu musical. À partir des années 1910, les enquêtes épistolaires "referendum » qui invitent les personnalités sondées à se positionner " pour ou contre " une nouveauté technologique (en particulier le disque), un courant controversé ou d'autres sujets sur lesquels on sait que les avis sont tranchés, peuvent entrainer des prises de positions radicales, parfois virulentes.

C'est le cas de l'enquête sur le jazz publiée par Paris-Midi en 1925 et dont la première question était : "Le jazz-band est-il pour vous "de la musique" ? ». Si les réponses

11 Le procédé rappelle clairement un compte rendu d'enquête de Huret qui se focalisait sur un accessoire similaire et s'achevait de la même façon : "Et M. Brunetière alluma enfin sa cigarette " (Carbonnel 2004a, note 32). Le tabac semble du reste un motif dans les entretiens de Schmitt. Déjà en 1911, Marcel Orban rapportait dans une entrevue avec le compositeur, après avoir décrit la maison et son propriétaire occupé à allumer un feu dans sa cheminée : «Je viens, lui-dis-je, recueillir vos impressions sur votre récent voyage en URSS. Mais Florent Schmitt parle de mille choses sauf de celle-là. Son feu, ses cigarettes, l'occupent tellement... Il se décide enfin » (Orban 1934, cité dans Duchesneau 2016, p. 171).

12 On pense ici aux passages descriptifs apposés aux sections dialoguées qui s'apparentent à des didascalies.

13 L'interview a été critiquée pour son style relâché à ses débuts (Campos 2016, p. 155). 
recueillies sont variées, certaines sont particulièrement violentes, comme celle de Jean Déré, compositeur et organiste, dont les propos cinglants s'accompagnent d'un discours antisémite et xénophobe ${ }^{14}$. Les journalistes peuvent également publier des propos outrageants de compositeurs sur d'autres compositeurs, comme on l'a vu avec Schmitt. Les attaques sont alors rarement ad hominem, même si les personnalités visées sont quelquefois identifiables. Le début de l'entretien de Vincent d'Indy avec Maudru en 1928 fait toutefois exception à la règle :

- Que pensez-vous d'Arnold Schoenberg?

- C'est un fou.

Je lève vers M. Vincent d'Indy des yeux exorbités. Le directeur de la Schola Cantorum émet ce jugement sans émotion, avec une aimable et tranquille indulgence, comme la constatation d'une évidente vérité.

- Pourtant, si nous énumérions tous les artistes qui se réclament de lui, tous ceux qui forment son école...

- Quels artistes ? Quelle école ? Schoenberg n'enseigne rien, sinon qu'il faut écrire tout ce qui passe par la cervelle. Ce n'est pas un enseignement : c'est une opinion, tout au plus ! Son œuvre, intéressante au début, n'est plus qu'un amas de notes sans signification. [...] Malgré cela, je comprends son succès. Une théorie qui recommande d'écrire n'importe quoi devait plaire aux nouvelles générations de musiciens ${ }^{15}$ (Maudru 1928d, p. 1-2).

L'enquêteur tire ici tout le profit d'un début d'entretien fracassant, d'autant plus qu'il joue lui-même l'effarouché en se montrant choqué par les propos de d'Indy. Voilà une bonne manière de lancer la polémique, ce qui ne manquera pas d'arriver puisque plusieurs compositeurs interrogés prendront la défense de Schoenberg et des jeunes générations suite à la publication de cet entretien.

L'enquête de Comoedia prend alors la tournure d'un débat arbitré par Maudru. C'est cependant Pierre Lalo, un des principaux critiques musicaux du début du siècle et grand pourfendeur de Ravel, qui est chargé de conclure l'enquête. Accusant comme à son habitude les jeunes d'être des " démolisseurs ", Lalo y condamne l'ensemble de la jeune musique, « de la menuaille ravélliste à la pierraille polytonale, en passant par des jazz plus ou moins nègres » (Lalo 1928). En proposant dix ans après la fin de la guerre de dresser le bilan et les perspectives de la création musicale, Maudru

14 «C'est la mort de l'intelligence... le règne de la bête... et ceci serait de l'art ! [...] Dans le jazz il n'y a que l'infini de la bêtise humaine. Il est vrai que cette sorte de vertige béat s'adapte admirablement à notre époque de crétinisme et à la veulerie de la plupart des individus. C'est la danse du veau d'or ! Le trémoussement de la mercante ! [...] Par crainte de ne pas être " dans le mouvement ", nous dédaignons les enseignements d'un Fauré, d'un Vincent d'Indy et nous allons demander aux nègres des recettes nouvelles... qui d'ailleurs seront démodés dans quelques années " (Couroy et Schaeffner 1925). Ce texte n'est pas sans rappeler « l'affaire des poisons » lancée deux ans plus tôt par un article de Louis Vuillemin qui s'attaquait aux « concerts métèques » de Jean Wiéner (Vuillemin 1923).

15 Mais d'Indy ne s'arrête pas là et continue d'épingler ses confrères lorsqu'on le questionne sur la création musicale à l'étranger : la musique de Korngold est pour lui « du Puccini avec des fausses notes »; quant à Varèse, il «n'a jamais trouvé une idée musicale de sa vie » (Maudru 1928d, p. 1-2). Sur les entretiens de d'Indy, voir l'étude de Gilles Saint-Arroman (2016). 
alimente ainsi les conflits qui opposent la jeune génération aux musiciens établis tout en favorisant ces derniers.

\section{LES STRATÉGIES DE L'ENQUÊTÉ}

Le sondage peut ainsi devenir un espace médiatique permettant aux compositeurs de ferrailler avec leurs pairs. Dans l'enquête de Comoedia, Schmitt et d'Indy font montre d'une singulière pugnacité. Fragilisés par la progressive reconnaissance de la génération d'après-guerre et par l'esprit des années 1920, les deux hommes recourent fréquemment à l'invective et aux attaques personnelles, mais aussi à l'ironie et à la provocation. Schmitt se présente ainsi comme un homme qui n'a rien à perdre ni à cacher :

Je vous autorise à publier tout ce que je dis là. J'ai tant d'ennemis, qu'il m'importe assez peu d'en compter dix de plus et dix de moins ! J'aimerais mieux avoir une allumette qui prenne !... Je ne compte plus sur l'avenir et je n'ai jamais eu la prudence comme ligne de conduite. Je refuse formellement de me mettre à plat ventre devant tous ces petits pompiers. Je trouve déplorable qu'un des plus grands musiciens de ma génération ait fait une démarche auprès des organisateurs du festival de Sienne ${ }^{16}$ pour leur recommander deux producteurs de ces inepties sur lesquelles on jette un coup d'œil, puis qu'on lance au panier (Maudru 1928f, p. 1-2).

Avant Schmitt, Gabriel Pierné, Henri Rabaud, d'Indy et Georges Hue avaient déjà critiqué l'école " de la fausse note ", la recherche de "la réclame et [de] la fausse gloire " (Maudru 1928e, p. 1), le manque de métier et de respect de la tradition des jeunes. Premier jeune compositeur interrogé, Arthur Honegger avait parlé de malentendu tout en défendant Schoenberg - attaqué comme on l'a vu par d'Indy (Maudru 1928c). Cette réponse avait suscité la réaction de d'Indy dans une «lettre ouverte à M. Honegger » (accompagnée d'un portrait du « Maître ») qui prenait un malin plaisir à humilier le jeune compositeur :

Mon cher Honegger,

Vous savez que je vous ai toujours trouvé très gentil, depuis le jour, déjà lointain, où je vous ai fourni l'occasion de diriger votre Mort du chef sauvage (je ne me rappelle plus le titre exact) à un exercice d'élèves, au Conservatoire. Je ne veux donc pas engager avec vous une polémique inutile entre nous, mais relever simplement quelques assertions de votre interview qui m'ont semblé la négation même de ce que vous prétendez prouver ( $\underline{\text { d'Indy 1928) }})^{17}$.

D'Indy rappelle ainsi avec condescendance son rôle de professeur bienveillant au Conservatoire tout en évoquant une œuvre de jeunesse d'Honegger, Le chant de Nigamon, qu'il a dirigée en 1918. En écrivant qu'il ne se souvient plus de son titre,

16 Il s'agit du festival de la Société internationale de musique contemporaine organisé à Sienne en 1928.

17 La «Lettre ouverte à M. Honegger » de Vincent d'Indy est publiée en une, comme toutes les réponses à l'enquête de Pierre Maudru. C'est cependant l'un des rares textes à être accompagné d'une photographie de l'auteur (présentée sur la page de façon tout à fait symétrique à celle de Victor Hugo, ce qui a pour effet de les mettre sur le même plan). 
d'Indy suggère qu'il s'agit d'une œuvre insignifiante, et le nom erroné qu'il lui attribue (La mort du chef sauvage) fait l'effet d'un pastiche de titre exotique ou primitiviste visant à dévaloriser son auteur. Mais d'Indy ne s'arrête pas là. Il commente ensuite avec sarcasme l'entretien d'Honegger, dont il reprend les points principaux, notamment la " libération de la tonalité » mentionnée par le compositeur suisse :

Alors il paraît que M. Schoenberg nous aurait apporté une « libération complète de la tonalité », et vous paraissez enchanté de cette ablation d'un membre vital de la musique...

Cela me fait l'effet d'un monsieur auquel on vient de couper les deux jambes et qui s'écrie : «Enfin, me voilà délivré de mes membres inférieurs! Désormais, je n'aurai plus jamais envie d'aller à pied ! » (d'Indy 1928)

L'analogie employée par d'Indy illustre bien sa conception essentialiste du langage musical : la tonalité est un "membre vital " de la musique et s'en débarrasser équivaudrait à une amputation des deux jambes entrainant l'impossibilité d'avancer et donc de créer. Si Honegger ne donne pas suite, du moins publiquement, à la lettre humiliante de d'Indy, André Messager déplore en revanche la tournure polémique prise par l'enquête et appelle à l'apaisement et à l'objectivité des parties. Mais quelques semaines plus tard, Maudru annonce que la réponse d'Alfred Bruneau « rouvre le débat que semblait clore la confiance généreusement exprimée par Messager à l'égard de la jeune école " (Maudru 1928g, p. 1). Bruneau, puis Ropartz, Pierre Vidal et Louis Aubert se rangent alors du côté de d'Indy et de Schmitt. Cependant Darius Milhaud, interrogé ensuite, se fait le porte-parole des jeunes et retourne l'accusation de manque de sérieux et d'expérience à ses adversaires :

Ceux qui nous accusent d'ignorer ou de méconnaître le passé, la tradition, sont de jeunes écervelés qui ne réfléchissent pas ou ne se donnent pas la peine d'étudier nos travaux.

- Mais savez-vous bien que parmi ceux-là, il y a des membres de l'Institut ?

M. Darius Milhaud sourit :

- Je ne me rétracte pas. Ces membres de l'Institut sont de jeunes écervelés, voilà tout !... Qu'ils se donnent la peine de lire attentivement les partitions d'Honegger, de Poulenc, d'Auric, de Prokofieff, de Stravinsky, d'Hindemith, de Webern, de Rieti, de Nabokoff... J'en passe et des meilleurs! (Maudru 1928a)

On le voit, ce sondage de Comodia révèle le conflit artistique et générationnel qui oppose alors l'avant-garde, et en particulier les jeunes, aux compositeurs de l'arrière-garde. L'enquête devient alors un lieu d'expression des luttes qui traversent le milieu musical, dévoilant les alliances et les divisions et entrainant de nouveaux débats. Le sondage se déployant souvent sur plusieurs semaines, voire plusieurs mois, il est fréquent que les derniers sondés tiennent compte des résultats publiés et viennent infirmer ou confirmer les propos des collègues interrogés précédemment. Les personnalités sondées participent ainsi à l'écriture collective d'un feuilleton à la forme indéterminée et dont la texture polyphonique s'enrichit au fur et à mesure des publications.

Les résultats d'enquête peuvent par ailleurs circuler d'un support à l'autre par leur reprise dans d'autres publications. Ces rééditions partielles ou résumés d'enquêtes 
(que l'on trouve en particulier dans les revues spécialisées) témoignent du succès et du crédit accordé aux sondages, mais aussi du déploiement de la parole du compositeur qui voit le nombre de ses lecteurs se multiplier dès lors que l'enquête est relayée par un autre media. Ces nouvelles publications ne peuvent que profiter aux compositeurs : la présence dans la presse est perçue comme un gage de popularité et de réussite et offre de potentielles perspectives dans le contexte tendu de la chute du mécénat après la Première Guerre mondiale.

Cela ne signifie pas pour autant que le compositeur interrogé par écrit réponde toujours aux questions posées. Il peut ainsi refuser de se prononcer et signifier à l'enquêteur que ses interrogations sont déplacées, trop complexes, ou qu'il ne souhaite pas entrer dans le débat. Ainsi Paul Dukas juge-t-il qu'il est trop tôt pour parler de l'avenir de la musique en 1915 :

Je suis très sensible à l'intérêt que vous voulez bien prendre à mes prévisions sur l'Avenir musical. Mais je ne puis que vous dire : toute discussion de cet ordre me semble prématurée et pour vous dire la vérité, la musique est la chose du monde à quoi j'ai le moins pensé depuis dix mois. Veuillez donc avoir l'amabilité de m'excuser pour l'instant (Anonyme 1915).

Dukas se présente ainsi, de façon discrète, comme un patriote dont l'esprit est tout entier accaparé par la guerre, une position partagée par nombre d'artistes en ce début de conflit. D'autres peuvent encore décider de ne pas répondre aux questions posées parce qu'ils se sont déjà exprimés ailleurs sur le sujet de l'enquête : c'est le cas de Camille Saint-Saëns qui renvoie en 1910 à un de ses articles que l'enquêteur décide de republier partiellement ([Pioch] 1910).

Les réponses des compositeurs peuvent aussi être laconiques et prendre une tournure désinvolte, en particulier à partir des années 1920. C'est le cas par exemple de Maxime Jacob répondant à l'enquête de la revue Musique en 1928 :

$1^{\circ}$ Mon modèle ? C'est moi-même.

Mes maîtres sont Bach, Mozart, Debussy et Erik Satie.

$2^{\circ}$ Je n'ai d'autre direction que celles de ma fantaisie, d'autres dogmes artistiques que ceux dictés par mon caprice.

Pôle d'attraction : Gounod.

Pôle de répulsion : Schönberg, Honegger, Florent Schmitt et, hélas, beaucoup d'autres (dans Honegger 1992, p. 94).

Près de vingt ans plus tard, Francis Poulenc écrira le même genre de réponses à l'emporte-pièce au critique Fred Goldbeck, neutralisant les questions techniques et croyant (ou feignant de croire) que ses réponses ne seront pas prises en compte :

Je ne comprends rien aux enquêtes.

Quand j'aurai dit à vos lecteurs :

(1) que mon " canon ", c'est l'instinct ;

(2) que je n'ai pas de principe et que je m'en vante ;

(3) que, Dieu merci ! je n'ai aucun système d'écriture (système équivalant pour moi à «trucs ») ; 
(4) que l'inspiration est une chose si mystérieuse qu'il vaut mieux ne pas l'expliquer, croyez-vous qu'ils seront transportés d'intérêt ? J'en doute. Ne comptez donc pas sur un laïus de moi $^{18} \ldots$ (Goldbeck 1946, p. 49)

On le voit, ces " prestes pirouettes », comme les qualifie Goldbeck, permettent à Poulenc de se mettre en avant mais aussi de dévaloriser le genre de l'enquête et les questions qui lui sont posées.

\section{L'ENQUÊTE SOUS LE FEU DES CRITIQUES}

Si elle attire nombre de journalistes et d'auteurs, l'enquête musicale ne fait pas l'unanimité dans la France des années 1910 à 1940. On critique en premier lieu son utilisation massive par des journalistes soupçonnés de chercher à " faire de la copie à peu de frais ». C'est ce que souligne Francis Rebékar, un facétieux journaliste du Ménestrel écrivant probablement sous pseudonyme, en août 1926 :

Voici les vacances, tout le monde s'en doute, n'est-ce pas, mais l'aspect de nos rues n'en serait-il pas une preuve que les Parisiens s'en apercevraient à la lecture des journaux : 1'ère des enquêtes est ouverte. L'enquête est un moyen sûr et périodique pour les journalistes à court d'événements de se procurer de la copie à peu de frais et surtout à peu de frais d'imagination : il fait si chaud qu'il vaut mieux laisser les autres se fatiguer, d'autant plus que ces autres ne sont pas fâchés de se rappeler au souvenir du public qui devant les flots, sur les pics ou sous les pins risquerait de les oublier ; si le journal qui reçoit les réponses veut bien accompagner celles-ci d'une photographie généralement rajeunie, le bonheur du questionné est complet (Rebékar 1926, p. 37).

Rebékar pointe ici avec humour la stratégie gagnant-gagnant qui motive cette publication à plusieurs voix. Il suggère aussi que les journalistes devraient être plus créatifs et ne pas se borner à faire parler les autres. Il remet ainsi en cause la pratique plus générale de l'interview qui inciterait à la paresse intellectuelle. L'enquête épistolaire et estivale, souvent dédiée aux "devoirs de vacances » des écrivains et des compositeurs (Duchesneau 2016, p. 173), fait 1'objet d'autres critiques narquoises, comme celle publiée en 1930 par Le Ménestrel : ici le journaliste vise en particulier les auteurs dramatiques et les compositeurs qui promettent une abondante production alors qu'ils ne disposent ni du temps ni des moyens pour écrire et monter dans l'année toutes les œuvres annoncées ${ }^{19}$.

18 En réalité la lettre se terminait ainsi : «Ne comptez donc pas sur un laïus de moi et croyez-moi votre Puck fidèle ». Goldbeck ne publie pas la fin de la phrase qui fait référence au surnom shakespearien qu'il donnait lui-même à Poulenc dans ses articles et qui n'aurait pas été comprise par les lecteurs de la revue (Poulenc 2011, p. 438).

19 «Plusieurs de nos confrères quotidiens demandent aux auteurs dramatiques et aux compositeurs quelles sont les œuvres qu'ils préparent et quels sont leurs projets pour l'an prochain. Cette enquête est annuelle : elle a l'avantage de procurer aux journalistes, veuf de tout événement, une copie abondante et que l'on passe selon les besoins de la mise en pages.

Ce qui nous frappe dans les résultats d'une telle enquête, c'est de voir combien les auteurs sont laborieux pendant la période que les autres appellent les vacances et quel labeur fécond ! S'il fallait écouler toute la production annoncée, le triple de nos théâtres parisiens serait insuffisant. 
Mais en 1926, Rebékar ironise surtout sur les sujets des enquêtes aux écrivains et aux acteurs, à qui l'on demande ce qu'ils préparent et comment ils passent leurs vacances, et s'étonne du fait qu'on ne sonde presque jamais les musiciens :

On ne demande presque jamais rien aux musiciens, et cependant ils prennent leurs vacances tout comme les autres et ne seraient peut-être pas fâchés qu'on parlât aussi un peu d'eux ou qu'on les fît parler ; ce n'est pas parce qu'ils écrivent généralement sur des portées de cinq lignes qu'ils ne sauraient trousser tout aussi élégamment une lettre que leurs confrères du théâtre ou du roman, et cependant Dieu sait que les sujets d'enquête sont multiples. En voici quelques-uns que je livre généreusement aux confrères :

$1^{\circ}$ Pourquoi la forme d'opéra telle que la pratiquait Meyerbeer ou Halévy est-elle tombée en désuétude?

$2^{\circ}$ D'où vient la vogue du drame lyrique? Verra-t-il aussi une décadence prochaine? $3^{\circ}$ Que préférez-vous, la musique dramatique ou la musique symphonique ? Et pourquoi?

$4^{\circ}$ Quelles sont les raisons de la faveur toujours croissante de l'opérette, etc., etc.

On pourrait agiter également la question des réformes de l'enseignement $d u$ Conservatoire, mais, pour épuiser le sujet, plusieurs périodes de vacances seraient nécessaires.

Aux techniciens, on pourrait demander leur avis sur les suites de quintes (de toux), sur l'utilisation des notes de passage (à l'hôtel), sur la tonalité, l'atonalité, la polytonalité, la moralité et l'amoralité, sur la chanteuse avec laquelle ils voudraient être fiancés, sur l'œuvre musicale qu'il serait souhaitable de voir exécuter le plus souvent, sur l'âge où ils ont commencé à reconnaître leurs notes, etc., etc. (Rebékar 1926, p. 376).

Comme on le voit, les sujets rebattus laissent rapidement place à des propositions ironiques et malicieuses qui pointent en réalité le caractère superficiel et dépourvu d'intérêt des enquêtes. Rebékar montre ici que si l'enquête vise à offrir au public des clés d'explications des pratiques artistiques de son temps, elle relève aussi d'un journalisme de vedettariat qui met en scène le créateur jusque dans sa vie privée, le rendant ainsi accessible au grand public.

De leur côté, les compositeurs se méfient souvent des enquêtes, par crainte de voir leurs propos déformés, mais aussi parce que les questions posées ne leur semblent pas toujours pertinentes ou légitimes. C'est le cas de Georges Auric, qui en avril 1940 publie un article dans Marianne pour discréditer l'enquête de Claude Chamfray intitulée "Y aura-t-il une musique de guerre ? (Chamfray 1939). Le compositeur, qui est peut-être aussi contrarié de n'avoir pas été consulté, déclare alors que la

Quant aux projets, ce sont surtout ceux des directeurs de théâtre dont les auteurs sont obligés de parler, car c'est de ceux-ci que dépend l'apparition des pièces. Si nous ne croyons les auteurs, les directeurs auraient fait de telles promesses... qu'on pourrait les prendre pour des députés ! N'est-il pas à craindre qu'ils ne fassent comme nos représentants et ne les tiennent pas?

Nous ne cueillerons rien dans ces enquêtes, car la feuille quotidienne passe, mais le périodique se garde, et si dans un an on se reportait à nos citations, combien des œuvres annoncées ou n'auraient pas vu le jour ou ne seraient même pas commencées ! Nous ne voulons pas mettre les auteurs en pénible situation » (Anonyme 1930). 
question est totalement inutile et qu'il est tout simplement impossible d'en débattre alors que le conflit mobilise l'ensemble de la communauté musicienne (Auric 1940). Si la réaction d'Auric est similaire à celle de Dukas en 1915 (voir plus haut), elle fait montre d'une remise en cause de la démarche de l'enquêteur (ici une enquêtrice) qui semble inexistante deux décennies plus tôt.

Comme le montre cet article d'Auric, le compositeur peut décider de prendre part à une discussion à laquelle il n'a pas été invité. En 1945, par exemple, Honegger réagit publiquement à la réponse d'Olivier Messiaen à une enquête sur le " désarroi musical » parue dans la revue Arts (Honegger 1945). Honegger commence par écrire tout ce que lui aurait répondu à cette enquête avant de reprendre point par point la réponse de Messiaen (qui a 15 ans de moins que lui) et de conclure :

Cher Messiaen, parlons maintenant sérieusement. Je crois que nous serons d'accord pour dire que les curieuses déclarations publiées dans Arts ne sont pas l'expression réelle de votre pensée. [...]

Il m'est arrivé souvent la même mésaventure. Nous oublions trop que la musique est un art dont les lois sont à la fois précises et mystérieuses. Qu'il est difficile d'en parler à ceux qui n'ont pas la même compréhension des termes techniques que nous employons.

Méfions-nous des enquêteurs. Ils nous écoutent avec beaucoup de condescendance, nous nous grisons de nos idées devant eux et le lendemain nous lisons avec épouvante, sinon un tissu de sottises, du moins un ensemble d'affirmations qui ne correspondent en rien à ce que nous avions cru exprimer ${ }^{20}$ (ibid., p. 5).

On voit bien ici que le temps et l'évolution des situations des compositeurs dans le milieu déplacent leurs rôles dans le champ médiatique. Honegger, jeune compositeur qui se faisait humilier par d'Indy en 1928 est en 1945 un musicien consacré, et sa position, bien que fragilisée au sortir de la guerre, lui permet de faire la leçon à Messiaen. Cet article d'Honegger montre également que la méfiance des compositeurs envers les enquêteurs, que Debussy accusait déjà en 1904 de déformer ses propos (Campos 2016, p. 165-166), se maintient au cours de la première partie du siècle.

$$
* * *
$$

S'ils acceptent volontiers de se prêter au jeu, les compositeurs des années 1910 à 1940 adoptent une attitude ambivalente à l'égard de l'enquête de presse : ils ont bien conscience qu'elle est un formidable outil de communication et de promotion, mais aussi, parce qu'elle implique la médiation d'un journaliste, un type d'écrit qui peut trahir leur pensée.

Alors que la presse de l'entre-deux-guerres ralentit sa diffusion et voit baisser le nombre de ses titres, l'enquête aux compositeurs continue d'être pratiquée, même si le rythme semble moins soutenu qu'avant 1914. Cependant, l'enquête-entretien semble

20 Honegger accuse ici les enquêteurs de modifier les propos tenus à l'oral, mais aussi de recomposer ce qui a été dit, et donc de trahir la pensée de l'auteur. 
progressivement délaissée au profit de l'enquête épistolaire, plus simple et rapide à réaliser et plus productive que la première. L'accélération des délais de production des journaux de même que le goût pour la collection d'opinions expliquent en grande partie cette évolution (Carbonnel 2004a, p. 40). Si au début du siècle le sondage oral ou écrit s'adressait à un petit cercle de musiciens consacrés, à partir des années 1920, l'enquête (qui est le plus souvent épistolaire) concerne de très nombreux compositeurs. Dans certains cas, le questionnaire est transmis non pas par correspondance mais par voie de presse, ce qui permet d'ouvrir l'enquête à des profils variés ainsi qu'aux amateurs $^{21}$. Toutefois l'enquêteur peut être accusé d'utiliser l'enquête pour faire de la copie, ce qui fragilise le crédit qu'on lui accordait auparavant.

Les enquêtes de l'entre-deux-guerres sont également révélatrices et productrices de conflits qui semblent s'exprimer de façon de plus en plus violente - certains propos montrent que l'on s'éloigne clairement de l'environnement policé du début du siècle. Ces sondages illustrent et nourrissent les luttes générationnelles et esthétiques des musiciens et corrélativement la partition académisme/avant-garde du milieu musical savant. Les enquêtes entrent par ailleurs dans des considérations plus techniques à la fin de la période, ce qui s'explique notamment par le développement de langages musicaux plus complexes et par la progressive spécialisation des journalistes. On peut enfin observer une timide politisation de cet espace médiatique qu'est l'enquête : les sondages des années 1914-1918 témoignaient de l'émergence de prises de positions qui se développeront de façon plus nette dans les années 1930-1940 et surtout au sortir des quatre années d'Occupation ${ }^{22}$. Plus difficile d'accès que celui de la période précédente, le corpus de ces deux décennies (qui inclut de nombreux journaux et revues politiques et culturelles) est encore mal connu et doit être comparé aux nouvelles formes d'enquêtes comme celles menées lors d'entretiens radiophoniques. L'enquête radiophonique, qui donne à entendre la voix-même de l'auteur, deviendra par la suite un nouveau modèle de sondage, comme en témoigne la célèbre enquête menée par Bernard Gavoty et Daniel-Lesur à la RTF auprès de 98 compositeurs dans leur émission : "Pour ou contre la musique moderne ${ }^{23}$ ?"

21 Cette forme de l'appel à contribution semble toutefois plus rare. Voir à ce sujet l'étude de Michel Duchesneau (2016, p. 171).

22 Sous l'Occupation l'enquête semble délaissée, ce qui peut s'expliquer par l'omniprésence de la censure et de l'auto-censure.

23 Ces entretiens ont ensuite été transcris et publiés sous forme d'un volume (Gavoty et Daniel-Lesur 1957). 
Anonyme (1915), "Les Théâtres. À l'Opéra " (réponse de Paul Dukas à une enquête), Le Cri de Paris, $19^{\mathrm{e}}$ année, $\mathrm{n}^{\circ} 957$ (1 $1^{\mathrm{er}}$ août), p. 11, https://gallica.bnf.fr/ark:/12148/bpt6k6523181p/f15.item, consulté le 5 janvier 2020.

Anonyme (1930), "Échos et nouvelles ", Le Ménestrel, 92e année, no 33 (15 août), p. 360, https://gallica.bnf.fr/ark:/12148/bpt6k56215535/f10.item, consulté le 5 janvier 2020.

Auric, Georges (1940), « La musique. Autour d'une enquête ", Marianne, 9e année, no 390 (10 avril), https://dicteco.huma-num.fr/fr/article/31584, consulté le 5 janvier 2020.

[Bender, Gabriel] (1932), «Une enquête. Les applaudissements au concert », Le Guide du concert, vol. 19, $\mathrm{n}^{\circ} 1$ (7 octobre), p. 14, http://1mhsbd.oicrm.org/media/public/documents/ART-BEG-1932-01. pdf, consulté le 5 janvier 2020.

Chamfray, Claude (1939), "Une enquête. Y aura-t-il une musique de guerre ? », La Revue musicale, vol. 20, nº 194 (décembre), p. 146-152.

Coeuroy, André, et André Schaeffner (1925), «Enquête sur le Jazz-Band, réponse de Mr. Jean Déré» , Paris-Midi, $15^{\mathrm{e}}$ année, $\mathrm{n}^{\circ} 60$ (29 mai), p. 3, https://gallica.bnf.fr/ark:/12148/bpt6k4735395c.item, consulté le 5 janvier 2020.

D’Indy, Vincent (1928), « Lettre ouverte à M. Honegger », Comoedia, 22 $2^{\mathrm{e}}$ année, $\mathrm{n}^{\circ} 5528$ (25 février), p. 1, https://gallica.bnf.fr/ark:/12148/bpt6k7651498n.item.r=comoedia, consulté le 5 janvier 2020.

Gavoty, Bernard, et Daniel-Lesur (1957), Pour ou contre la musique moderne, Paris, Flammarion.

Goldbeck, Fred (1946), "Francis Poulenc, ou les prestes pirouettes ", Contrepoints, n ${ }^{\circ} 1$ (janvier), p. 49, repris dans Poulenc 2011, p. 437-438.

Honegger, Arthur (1945), «Sur le désarroi musical », $X X^{\circ}$ siècle, $1^{\text {ère }}$ année, $n^{\circ} 7$ (8 novembre), p. 1 et 5 , repris dans Honegger 1992, p. 181-183.

Honegger, Arthur (1992), Écrits, textes réunis et annotés par Huguette Calmel, Paris, Champion.

Lalo, Pierre (1928), "Conclusion sur une enquête de Comoedia. La musique d'aujourd'hui », Comoedia, $22^{\mathrm{e}}$ année, $\mathrm{n}^{\circ} 5635$ (11 juin), p. 1 ; n 5636 (12 juin), p. 1, https://gallica.bnf.fr/ark:/12148/ cb32745939d/date1928.item, consulté le 5 janvier 2020.

Maudru, Pierre (1928a), « Défense des Jeunes et misère des théâtres d'État... selon Darius Milhaud », Comoedia, 22 ${ }^{\mathrm{e}}$ année, $\mathrm{n}^{\circ} 5620$ (26 mai), p. 1, https://gallica.bnf.fr/ark:/12148/bpt6k7651588m. item.r $=\mathrm{Com} \% \mathrm{C} 5 \% 93$ dia $\% 2 \mathrm{C}+7+$ avril +1928 , consulté le 5 janvier 2020 .

Maudru, Pierre(1928b), «Où en est la musique française?», Comoedia, $22^{\circledR}$ année, n 5514(11 février)p. 1-2, https://gallica.bnf.fr/ark:/12148/bpt6k7651484m.item.r=Com\%C5\%93dia $\% 2 \mathrm{C}+7+$ avril+1928, consulté le 5 janvier 2020.

Maudru, Pierre(1928c), «Où en estla musique française?», Comoedia, $22^{e}$ année, $n^{\circ} 5526$ (23 février)p. 1-2, https://gallica.bnf.fr/ark:/12148/bpt6k7651496t.item.r=Com\%C5\%93dia\%2C+7+avril+1928, consulté le 5 janvier 2020.

Maudru, Pierre (1928d), "Promenades et visites. Où en est la musique française ? ", Comoedia, $22^{\mathrm{e}}$ année, $\mathrm{n}^{\circ} 5524$ (21 février), p. 1 et 2, https://gallica.bnf.fr/ark:/12148/bpt6k76514940.item.

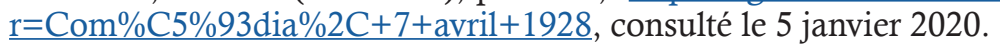

Maudru, Pierre (1928e), "Promenades et visites. Où en est la musique française ? ", Comoedia, $22^{\mathrm{e}}$ année, $\mathrm{n}^{\circ} 5531$ (28 février), p. 1 et 2, https://gallica.bnf.fr/ark:/12148/bpt6k7651501p.item.

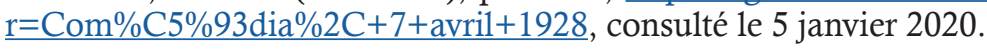

Maudru, Pierre (1928f), "Promenades et visites. Où en est la musique française ? ", Comøedia, $22^{\mathrm{e}}$ année, $\mathrm{n}^{\circ} 5554$ (22 mars), p. 1 et 2, https://gallica.bnf.fr/ark:/12148/bpt6k7651524p.item. $\underline{r}=\mathrm{Com} \% \mathrm{C} 5 \% 93 \mathrm{dia} \% 2 \mathrm{C}+7+$ avril +1928 , consulté le 5 janvier 2020 .

Maudru, Pierre (1928g), "Promenades et visites. Où va la musique moderne ? ", Comoedia, $22^{\mathrm{e}}$ année, $\mathrm{n}^{\mathrm{o}} 5574$ (12 avril), p. 1 et 2, https://gallica.bnf.fr/ark:/12148/bpt6k7651544f.item.

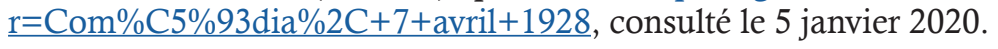


Maudru, Pierre (1928h), «Promenades et visites. Sur la musique gaie et l'opérette française », Comœedia, $22^{\mathrm{e}}$ année, $\mathrm{n}^{\circ} 5570$ (7 avril), p. 1 et 2, https://gallica.bnf.fr/ark:/12148/bpt6k7651540s/f1.item. r=Com\%C5\%93dia, \%207\%20avril\%201928, consulté le 5 janvier 2020 .

Orban, Marcel (1934), "L'U.R.S.S. vue par Florent Schmitt », Le Guide du concert, vol. 21, n 6 (9 novembre), p. 138-140.

[Pioch, Georges] (1910), «Enquête sur la décadence de l'opéra-comique », Musica, nº 96 (septembre), p. 131-132.

Poulenc, Francis (2011), J'écris ce qui me chante, textes et entretiens réunis, présentés et annotés par Nicolas Southon, Paris, Fayard.

Rebékar, Francis (1926), "Variétés. Enquêtes », Le Ménestrel, 88 année, no 35 (27 août), p. 376, https://gallica.bnf.fr/ark:/12148/bpt6k5618093v/f10.item, consulté le 5 janvier 2020.

Vuillemin, Louis (1923), « Notes sans mesure. Concerts métèques », Le Courrier musical, $25^{\mathrm{e}}$ année, $\mathrm{n}^{\circ} 1$ ( $1^{\text {er }}$ janvier), p. 4.

\section{BIBLIOGRAPHIE}

Branger, Jean-Christophe (2011), «La réponse de Debussy à une enquête du Cri de Paris pendant la Grande Guerre », Cahiers Debussy, n 35, p. 97-108.

Brogniez, Laurence, et Valérie Dufour (dir.) (2016), Entretiens d'artistes. Poétique et pratiques, Paris, Vrin.

Campos, Rémy (2016), " L'interview de compositeur. Les débuts d'un genre biographique (18801930) », dans Brogniez et Dufour 2016, p. 151-168.

Carbonnel, Marie (2004a), «Le grand spectacle de la littérature. Réflexions autour de trois enquetes génériques ", dans Sylvie Triaire, Marie Blaise et Marie-Ève Thérenty (dir.), L’interview d'écrivain. Figures bibliques d'autorité, Montpellier, Presses universitaires de la Méditerranée, p. 251-275, https://books.openedition.org/pulm/324?lang=fr, consulté le 15 juillet 2019.

Carbonnel, Marie (2004b), « Les écrivains en leur miroir. Jeu et enjeux de l'enquête au sein de la République des Lettres ", Mil neuf cent. Revue d'histoire intellectuelle, 2004/1 n ${ }^{\circ}$ 22, p. 29-58, https://www.cairn.info/revue-mil-neuf-cent-2004-1-page-29.htm, consulté le 15 juillet 2019.

Duchesneau Michel (2016), «Entrevues de musiciens dans la presse musicale française. L'ère des "interventions médiatiques" (1900-1939) », dans Brogniez et Dufour 2016, p. 169-185.

Esner, Rachel (2013), "In the Artist's Studio with L'Illustration », RIHA Journal, $\mathrm{n}^{\circ} 0069$ (18 mars), http://www.riha-journal.org/articles/2013/2013-jan-mar/esner-lillustration, consulté le 10 décembre 2019.

Haug, Benoit (2010), " Travail de recherche sur les enquêtes et entrevues avec des musiciens dans la presse française de la première moitié $d u \mathrm{XX}^{e}$ siècle ", document disponible auprès de l'orcRM, Montréal.

Lazzaro, Federico (2017), "Chanter l'athlète moderne, entre ridiculisation et glorification ", Revue musicale oIcRM, vol. $4, \mathrm{n}^{\circ} 2$ (novembre), mis en ligne le 14 décembre 2017, http://revuemusicaleoicrm.org/rmo-vol4-n2/athlete-moderne/, consulté le 15 juillet 2019.

Saint-Arroman, Gilles (2016), «Vincent d'Indy au parloir. Usages et fiabilité de la transcription d'entretien au début du Xx ${ }^{e}$ siècle ", dans Brogniez et Dufour 2016, p. 49-60.

Seillan, Jean-Marie (2011), "L'interview. Définition, genèse, histoire ", dans Dominique Kalifa et al. (dir.), La civilisation du journal. Histoire culturelle et littéraire de la presse française au XIX siècle, Paris, Nouveau Monde éditions.

Thérenty, Marie-Ève (2009), « Paroles, paroles, paroles... Du portrait littéraire à l'interview d'écrivain » dans Marie Blaise, Sylvie Triaire et Alain Vaillant (dir.), L'histoire littéraire des écrivains. Paroles vives, Montpellier, Presses universitaires de la Méditerranée, p. 259-273, https://books.openedition.org/ pulm/855, consulté le 15 juillet 2019. 